\title{
Hemodynamic Features of Advanced Cirrhosis Due to Chronic Bile Duct Ligation
}

\author{
Yasumi Katsuta', Xue-Jun Zhang ${ }^{1}$, Masaru Ohsuga', Toshio Akimoto', \\ Hirokazu Komeichi', Shuji Shimizu', Toru Inami', Akiko Miyamoto', \\ Katsuaki Satomura ${ }^{1}$ and Teruo Takano ${ }^{1}$ \\ ${ }^{1}$ First Department of Internal Medicine, Nippon Medical School \\ ${ }^{2}$ Division of Laboratory Animal Science, Nippon Medical School
}

\begin{abstract}
Aim: The aim of the present study was to compare the hemodynamic features of portal hypertension in rats with early cirrhosis with those of rats with advanced cirrhosis following common bile duct ligation (CBDL).

Methods: A total of 53 male Sprague-Dawley rats were used. Hemodynamics were evaluated under conscious and unrestrained conditions 4 weeks and 8 weeks after CBDL, and 4 weeks after a sham operation. Arterial pressure and portal pressure were measured directly via catheters placed in the right femoral artery and main portal vein, respectively. The cardiac index and organ (splanchninc organs, brain, kidneys and lungs) blood flow were determined by the reference sample method using ${ }^{141} \mathrm{Ce}$-labeled microspheres $(15 \mu \mathrm{m}$ in diameter). Arterial levels of endothelin-1 and nitrate/nitrite, as well as liver function variables, were also determined.

Results: Portal pressure was significantly higher 8 weeks after CBDL $(15.8 \pm 2.1, \mathrm{n}=8)$ than 4 weeks after CBDL $(13.9 \pm 2.1 \mathrm{mmHg}, \mathrm{n}=12, \mathrm{p}<0.05)$, and the hyperdynamic circulation of the early period was attenuated $(\mathrm{p}<0.05)$. Although hepatic artery blood flow 4 and 8 weeks after CBDL was higher than that after sham operation $(\mathrm{p}<0.05)$, portal territory blood flow was not increased. There was a significant positive correlation between portal pressure and portal territory blood flow 8 weeks after CBDL $(r=0.822, n=8, p=0.012)$. In rats with anemia 4 weeks after $\mathrm{CBDL}$, the hemoglobin concentration was negatively correlated with portal territory blood flow $(r=-0.597, \mathrm{n}=12, \mathrm{p}=0.040)$.

Conclusion: Portal pressure was higher 8 weeks after CBDL than 4 weeks after CBDL and increased with portal territory blood flow, suggesting that portal hypertension is maintained by a mechanism consistent with the forward flow theory. Anemia might exacerbate the hyperdynamic systemic circulation 4 weeks after CBDL.
\end{abstract}

(J Nippon Med Sch 2005; 72: 217-225)

Key words: forward flow theory, portal hypertension, anemia, endothelin-1, nitrate

Correspondence to Yasumi Katsuta, MD, First Department of Internal Medicine, Nippon Medical School, 1-1-5 Sendagi, Bunkyo-ku, Tokyo 113-8603, Japan

E-mail: ykatsuta@nms.ac.jp

Journal Website (http://www.nms.ac.jp/jnms/) 


\section{Introduction}

Both common bile duct ligation (CBDL) and partial ligation of the portal vein (PVL) are widely used to create animal models of portal hypertension ${ }^{1-10}$. The major difference between these two models is that the former features intrahepatic portal hypertension with cirrhosis, whereas the latter shows a prehepatic portal hypertension without hepatic dysfunction. The maximal lifespan of rats after chronic bile duct ligation is limited to 6 weeks because of progressive liver damage due to complete obstruction of the biliary tract $^{3}$. We have investigated this animal model and have found that massive internal hemorrhage is the direct cause of death $^{3}$. In the setting of chronic bile duct obstruction, vitamin $\mathrm{K}$ deficiency could impair hemostasis and lead to such a massive internal hemorrhage. In fact, we can extend the survival of rats beyond 8 weeks after CBDL by providing a diet enriched with vitamins and nutrients ${ }^{3}$.

A hyperdynamic circulatory state associated with decreased systemic and splanchnic vascular resistance, arterial hypotension, and increased cardiac output is the hemodynamic pattern seen both in patients with advanced cirrhosis and in animal models of portal hypertension ${ }^{4,11-15}$. The mechanism underlying these circulatory abnormities is arteriolar vasodilatation, which particularly affects the splanchnic circulation ${ }^{13-15}$. The onset of portal hypertension and the persistence of chronic portal hypertension after the development of portal collaterals have been explained by the "backward flow" theory and the "forward flow" theory, respectively ${ }^{4,14-17}$. Animal models of portal hypertension due to CBDL are usually tested within 6 weeks after the procedure. To our knowledge, hemodynamics have never been studied beyond 8 weeks after CBDL in rats. The aim of the present study was to compare hemodynamic features between rats with advanced liver cirrhosis due to CBDL and rats with the early-stage cirrhosis after ligation by examining conscious and unrestrained animals.

\section{Materials and Methods}

\section{(1) Portal Hypertension Models}

A total of 53 male Sprague-Dawley rats weighing 200 to $250 \mathrm{~g}$ were used for the experiment. Bile duct ligation was carried out in 37 rats with a sterile procedure under anesthesia with ether and intraperitoneal pentobarbital sodium $(50 \mathrm{mg} / \mathrm{kg}$ ). The common bile duct was carefully exposed and double-ligated with silk threads, after which a 2-mm segment was resected between the ligatures to prevent recanalization. Sham surgery, involving mobilization of the common bile duct without ligation or resection, was performed in 16 rats in a similar manner. Twelve CBDL rats were studied 4 to 5 weeks after surgery (CBDL-4wk group), and 8 CBDL rats were studied 8 to 9 weeks after surtery (CBDL-8wk group). Eight Sham rats were studied with the CBDL-4wk group. After the experiments, the animals were killed with an overdose of intravenous pentobarbital sodium, and autopsy was carried out to macroscopically confirm the development of liver cirrhosis. To measure serum nitrate/nitrite (NOx) and plasma endothelin-1 (ET-1) levels and to perform liver function tests, a blood sample $(6$ to $8 \mathrm{ml})$ was drawn from the abdominal aorta after laparotomy was performed under ether anesthesia in 8 Sham rats, 8 CBDL- 4 wk rats, and 9 CBDL-8wk rats, which were then killed by exsanguination. Serum or plasma was separated from the blood (by centrifugation at 2,000 $g$ for 10 min at $4^{\circ} \mathrm{C}$ ) within 20 min of collection with careful handling to avoid hemolysis, and was stored at $-80^{\circ} \mathrm{C}$ until analysis.

\section{(2) Preparations for the Hemodynamic Study}

Insertion of catheters for hemodynamic measurements was done on the day before the experiment under the same anesthesia as that described above. All catheters were tunneled subcutaneously and exteriorized at the back of the neck. The animals were allowed to recover for 24 hours and then were fasted overnight with free access to water. Before the experiments, the animals were allowed to rest for at least 30 minutes after 
attachment of the experimental apparatus until they reached a physiologically stable condition, as confirmed by minimal fluctuation of arterial pressure with a stable heart rate and/or minimum body motion. Then hemodynamic measurements were performed in a quiet, air-conditioned room maintained at $24^{\circ} \mathrm{C}$, with the rats in a conscious and unrestrained state.

\section{(3) Hemodynamic Measurements}

Mean arterial pressure (MAP) and portal pressure were directly measured via catheters placed in the right femoral artery and at the bifurcation of the superior mesenteric and splenic veins, respectively. The cardiac index (CI) was determined by the reference sample method using ${ }^{141} \mathrm{Ce}-\mathrm{labeled}$ microspheres with a diameter of $15 \mu \mathrm{m}\left(\mathrm{NEN}^{\mathrm{TM}} \mathrm{Life}\right.$ Science Products, Inc. Boston, MA, U.S.A. ${ }^{5.6}$. In brief, $100 \times 10^{4}$ radiolabeled microspheres suspended in 0.8 $\mathrm{m} l$ of saline that contained $10 \%$ Ficoll-70 were gradually injected over $60 \mathrm{sec}$ through a silastic catheter placed in the left ventricle, and a $0.8-\mathrm{m} l$ reference blood sample was drawn simultaneously at a constant speed over $60 \mathrm{sec}$ via a catheter placed in the abdominal aorta using a Harvard medical pump (Model 2400-003, Harvard Apparatus Ltd., Holliston, MA, U.S.A.). The radioactivity of the preinjection microspheres, reference blood sample, and each organ was counted with a gamma-counter (Aloka 505, Aloka Ltd., Tokyo, Japan) at a setting of 500 to $650 \mathrm{keV}$ for the ${ }^{141} \mathrm{Ce}$ channel, and the results were corrected using a ${ }^{141} \mathrm{Ce}$ standard. Adequate mixing of microspheres was confirmed by a difference of $<10 \%$ between the left and right kidneys.

Calculations $^{6}$ : Hemodynamic parameters for the systemic and portal circulations were calculated with the following equations.

$\mathrm{CI}(\mathrm{ml} / \mathrm{min} / 100 \mathrm{~g}$ body weight $[\mathrm{BW}])=$ [injected radioactivity $(\mathrm{cpm}) /$ reference sample radioactivity $(\mathrm{cpm})] \times[100 / \mathrm{BW}(\mathrm{g})] \times 0.8(\mathrm{ml} / \mathrm{min})$.

Organ blood flow $(\mathrm{ml} / \mathrm{min} / 100 \mathrm{~g}$ BW $)=$ [organ radioactivity $(\mathrm{cpm}) /$ injected radioactivity $(\mathrm{cpm})] \times$ CI.

Systemic vascular resistance $(\mathrm{SVR})=\mathrm{MAP} \times 80 /$ CI (dynes $\cdot \mathrm{sec} \cdot \mathrm{cm}^{-5} / 100 \mathrm{~g} \mathrm{BW}$ ).

Portal territory blood flow (PTBF) was defined as the sum of the blood flow through the stomach, intestine, colon, pancreas, and mesentery.

Portal territory vascular resistance $($ PTVR $)=$ (MAP-Portal pressure) $\times 80 / \mathrm{PTBF}($ dynes $\cdot \mathrm{sec} \cdot$ $\left.\mathrm{cm}^{-5} / 100 \mathrm{~g} \mathrm{BW}\right)$.

Hepatic artery vascular resistance $=\mathrm{MAP} \times 80 /$ $\mathrm{HABF}$ (dynes $\cdot \mathrm{sec} \cdot \mathrm{cm}^{-5} / 100 \mathrm{~g} \mathrm{BW}$ ), where BW is body weight, and HABF is hepatic artery blood flow.

Hepatocollateral vascular resistance $(\mathrm{HCVR})=$ Portal pressure $\times 80 / \mathrm{PTBF}$ dynes $\cdot \mathrm{sec} \cdot \mathrm{cm}^{-5} / 100 \mathrm{~g}$ BW.

\section{(4) Laboratory Tests}

NOx and liver function tests

Serum samples for determination of NOx were deproteinized in ethanol and analyzed by an automated high performance liquid chromatography system according to the method of Green et al. ${ }^{18}$. In brief, a $15-\mu l$ aliquot of treated serum was injected into the system, nitrite and nitrate were separated by a reverse-phase column (IC-I 524 A; Showadenko Co., Tokyo, Japan), and nitrate was successively reduced to nitrite using a reduction column $(\mathrm{Cd}-\mathrm{Cu}$ reduction column; Tokyo Kasei Co., Tokyo, Japan). Then the nitrite (including that derived from nitrate) was mixed with Griess reagent (Tokyo Kasei Co.) and was analyzed at $540 \mathrm{~nm}$ using a visible-light spectrophotometer (SPD-10A; Shimazu Co., Tokyo, Japan). Standard liver function tests were performed with an autoanalyzer (Model 7170, Hitachi High-technologies, Co., Tokyo, Japan).

Plasma ET-1 level

A 2-m $l$ sample of arterial plasma containing EDTA-2Na and aprotinin was mixed with an octadecylsilyl silica particle suspension, and ET-1 was adsorbed to the particles ${ }^{19}$. Then the particles were separated from the plasma by centrifugation and ET-1 was eluted out in an aqueous solution of $60 \%$ methanol. This solution was evaporated to dryness in a nitrogen gas chamber and the residue was dissolved in the assay buffer (Tris buffer at $\mathrm{pH}$ 8.0 containing $0.1 \%$ human albumin, 0.01 M EDTA$2 \mathrm{Na}$, Tween $20,0.01 \%$ sodium azide, and $500 \mathrm{kIU} / \mathrm{ml}$ of aprotinin). Then ET-1 was measured with a double-antibody radioimmunoassay technique. Rabbit anti-ET-1 antibody was obtained from Peninsula (St. 
Table 1 Laboratory data of the three groups

\begin{tabular}{l|ccrc}
\hline & Sham rats $(\mathrm{n}=8)$ & CBDL-4wk $(\mathrm{n}=8)$ & CBDL-8wk $(\mathrm{n}=9)$ & P value \\
\hline Body weight $(\mathrm{g})$ & $341 \pm 15$ & $328 \pm 36.4^{\mathrm{b}}$ & $370 \pm 34^{\mathrm{c}}$ & $<0.02$ \\
Total bilirubin $(\mathrm{mg} / \mathrm{d} l)$ & $0.0 \pm 0.0^{\mathrm{a}}$ & $8.5 \pm 1.2^{\mathrm{b}}$ & $6.3 \pm 1.4^{\mathrm{c}}$ & $<0.001$ \\
ALT $(\mathrm{IU} / \mathrm{L})$ & $40.8 \pm 5.3^{\mathrm{a}}$ & $205.3 \pm 77.7$ & $213.9 \pm 62.0^{\mathrm{c}}$ & $<0.001$ \\
ALP $(\mathrm{IU} / \mathrm{L})$ & $485.6 \pm 57.0^{\mathrm{a}}$ & $1,496.3 \pm 386.1^{\mathrm{e}}$ & $1,819.6 \pm 372.5^{\mathrm{c}}$ & $<0.001$ \\
Albumin $(\mathrm{g} / \mathrm{d} l)$ & $4.0 \pm 0.1^{\mathrm{d}}$ & $3.7 \pm 0.2^{\mathrm{b}}$ & $3.1 \pm 0.3^{\mathrm{c}}$ & $<0.001$ \\
NOx $(\mu \mathrm{mol} / l)$ & $12.9 \pm 1.2$ & $17.5 \pm 3.8$ & $19.2 \pm 6.5^{\mathrm{c}}$ & $<0.01$ \\
Endothelin-1 $(\mu \mathrm{mol} / l)$ & $1.76 \pm 0.65^{\mathrm{a}}$ & $9.45 \pm 1.48^{\mathrm{b}}$ & $14.09 \pm 2.49^{\mathrm{c}}$ & $<0.001$ \\
\hline
\end{tabular}

Mean \pm SD.

Tukey's test (Spjotvoll/Stoline test for unequal sample sizes): ${ }^{\text {a }}$ p $<0.05$ vs. CBDK-4wk; ${ }^{\text {, }}$ p $<0.05$ vs. CBDL-8wk; c, $\mathrm{p}<0.05$ vs. Sham.

Fisher's PLSD test: ${ }^{\mathrm{d}}$, $\mathrm{p}<0.05$ vs. CBDL-4wk; ${ }^{\mathrm{e}}, \mathrm{p}<0.05$ vs. CBDL-8wk.

Helens, UK), $\left.{ }^{[25} \mathrm{I}\right]$-ET-1 was obtained NEN Life Science Products, Inc. (Boston, MA, U.S.A.), and human ET-1 was purchased from the Peptide Institute Inc. (Osaka, Japan).

\section{(5) Statistical Analysis}

Statistical analysis of differences between groups was done with one-way ANOVA with post hoc comparison using Tukey's test (or the Spjotvoll/ Stoline test for unequal sample sizes) or Fisher's PLSD test, as well as linear correlation analysis, using "Statistica” software (StatSoft Inc. Tulsa, OK. U.S.A.). All results are expressed as the mean \pm SD. Statistical significance was defined as $\mathrm{p}<0.05$. The study protocol was reviewed and approved by the Ethics Committee for Animal Experiments of Nippon Medical School.

\section{Results}

\section{(1) Laboratory Data}

Liver function tests revealed the features of cholestatic hepatic dysfunction in both CBDL groups (Table 1). The serum albumin level was significantly lower at 8 weeks after CBDL than 4 weeks after CBDL. Serum NOx levels were higher in both CBDL groups than in Sham rats. However, there was no significant difference in NOx levels between the CBDL- 8 wk rats and CBDL-4wk rats $(p=0.056)$. The plasma level of endothelin-1 was highest in the CBDL-8wk rats and lowest in the Sham rats, and the differences among the three groups were significant.

\section{(2) Hemodynamics}

Each variables of the systemic and splanchnic hemodynamics in rats with portal hypertension and Sham rats is listed in the Table 2. Hyperdynamic changes in the circulation, with an increased CI, decreased MAP, and reduced systemic vascular resistance, were significant in CBDL-4wk rats compared with those in Sham rats. In CBDL-8wk rats, however, MAP was similar to that in Sham rats and the difference in CI was not significant $(p=$ 0.085), although systemic vascular resistance was still significantly lower. Portal pressure was highest in CBDL-8wk rats. There were no significant differences in PTBF among the three groups. However, PTVR was lowest in the CBDL-4wk rats. HABF was significantly higher and HAVR was significantly lower in both CBDL groups than in Sham rats. HCVR was significantly higher in both CBDL groups than in Sham rats, and the difference between the two CBDL groups was also significant. The hemoglobin concentration of CBDL-4wk rats was significantly lower than that of Sham rats or CBDL-8wk rats.

\section{(3) Relationship among Hemodynamic Parameters}

There was a significant positive correlation and negative correlation between portal pressure and PTBF or PTVR, respectively in CBDL-8wk rats (Fig. 1), but no such correlations were found in CBDL-4wk rats or Sham rats. Although an 
Table 2 Systemic and splanchnic hemodynamics of the three groups

\begin{tabular}{|c|c|c|c|c|}
\hline & Sham rats $(\mathrm{n}=8)$ & CBDL-4wk $(\mathrm{n}=12)$ & CBDL 8wk $(\mathrm{n}=8)$ & $P$ value \\
\hline Body weight $(\mathrm{g})$ & $361.4 \pm 15.4^{\mathrm{a}}$ & $304.6 \pm 23.3^{b}$ & $356.3 \pm 35.3$ & $<0.001$ \\
\hline Hemoglobin $(\mathrm{g} / \mathrm{d} l)$ & $13.1 \pm 0.8^{\mathrm{a}}$ & $10.2 \pm 1.3^{\mathrm{b}}$ & $12.0 \pm 1.4$ & $<0.001$ \\
\hline \multicolumn{5}{|l|}{ Systemic hemodynamics: } \\
\hline Heart rate (bpm) & $346 \pm 28$ & $373 \pm 35^{\mathrm{e}}$ & $416 \pm 41^{c}$ & $<0.01$ \\
\hline MAP (mmHg) & $108.1 \pm 5.9^{\mathrm{a}}$ & $96.3 \pm 6.1^{\mathrm{b}}$ & $105.0 \pm 10.0$ & $<0.001$ \\
\hline $\mathrm{CI}(\mathrm{ml} / \mathrm{min} / 100 \mathrm{~g} \mathrm{BW})$ & $28.3 \pm 2.9^{\mathrm{a}}$ & $36.8 \pm 3.1^{\mathrm{b}}$ & $30.4 \pm 5.6$ & $<0.001$ \\
\hline $\mathrm{SVR}^{\mathrm{g}}$ & $307 \pm 31^{\mathrm{a}}$ & $211 \pm 22^{\mathrm{b}}$ & $283 \pm 52$ & $<0.001$ \\
\hline \multicolumn{5}{|l|}{ Splanchnic hemodynamics: } \\
\hline Portal pressure $(\mathrm{mmHg})$ & $6.2 \pm 1.3^{\mathrm{a}}$ & $13.9 \pm 2.1^{\mathrm{b}}$ & $15.8 \pm 2.1^{\mathrm{c}}$ & $<0.001$ \\
\hline PTBF $(\mathrm{ml} / \mathrm{min} / 100 \mathrm{~g} \mathrm{BW})$ & $7.2 \pm 0.7$ & $7.6 \pm 1.5$ & $6.4 \pm 1.9$ & $=0.498$ \\
\hline PTVR $^{g}$ & $1,141 \pm 87^{\mathrm{d}}$ & $904 \pm 237$ & $1,194 \pm 339$ & $<0.05$ \\
\hline HABF $(\mathrm{ml} / \mathrm{min} / 100 \mathrm{~g} \mathrm{BW})$ & $0.34 \pm 0.11^{\mathrm{a}}$ & $1.89 \pm 0.98$ & $1.93 \pm 0.87^{\mathrm{c}}$ & $<0.001$ \\
\hline $\mathrm{HAVR}^{\mathrm{g}}$ & $27,874 \pm 8,606^{\mathrm{a}}$ & $5,112 \pm 2,461$ & $5,498 \pm 3,348^{c}$ & $<0.001$ \\
\hline $\mathrm{HCVR}^{\mathrm{g}}$ & $70.1 \pm 18.3^{\mathrm{a}}$ & $150.5 \pm 31.8^{\mathrm{b}}$ & $208.8 \pm 51.6^{c}$ & $<0.001$ \\
\hline $\mathrm{RABF}(\mathrm{ml} / \mathrm{min} / 100 \mathrm{~g} \mathrm{BW})$ & $4.2 \pm 0.7^{\mathrm{a}}$ & $6.7 \pm 0.8$ & $6.3 \pm 1.1^{\mathrm{c}}$ & $<0.001$ \\
\hline $\mathrm{RAVR}^{\mathrm{g}}$ & $2,092 \pm 296^{\mathrm{a}}$ & $1,158 \pm 141$ & $1,375 \pm 208^{c}$ & $<0.001$ \\
\hline
\end{tabular}

Mean \pm SD. MAP, mean arterial pressure; CI, cardiac index; SVR, systemic vascular resistance; PTBF, portal territory blood flow; PTVR, portal territory vascular resistance; HABF, hepatic artery blood flow; HAVR, hepatic artery vascular resistance; RABF, renal artery blood flow; RAVR, renal artery vascular resistance; BW, body weight; ${ }^{\mathrm{g}}$, dynes $\cdot \mathrm{sec} \cdot \mathrm{m}^{-5} / 100 \mathrm{~g}$ body weight.

Tukey's test (Spjotvoll/Stoline test for unequal sample sizes): ${ }^{\text {a }}$ p $<0.05$ vs. CBDK- 4 wk; ${ }^{b}, \mathrm{p}<0.05$ vs. CBDL8 wk; ${ }^{c}, \mathrm{p}<0.05$ vs. Sham.

Fisher's PLSD test: ${ }^{\mathrm{d}}, \mathrm{p}<0.05$ vs. CBDL-4wk; ${ }^{\mathrm{e}}, \mathrm{p}<0.05$ vs. CBDL-8wk).
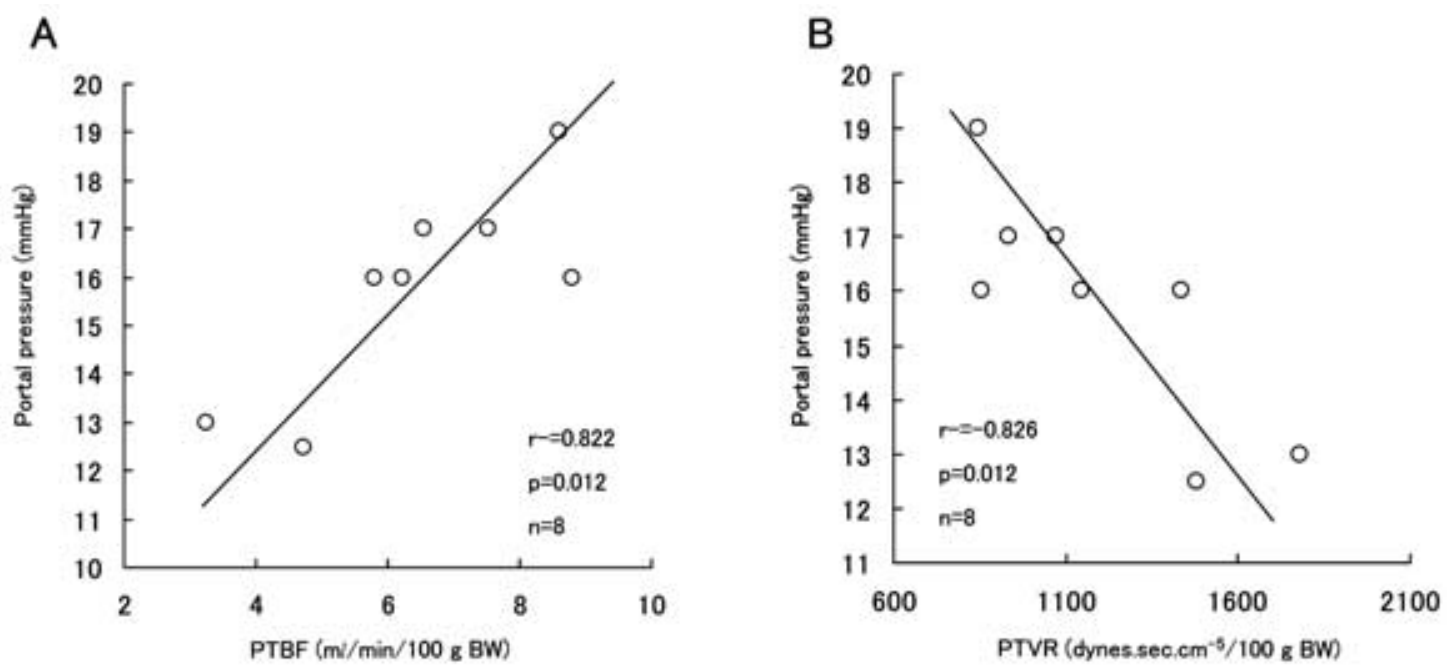

Fig. 1 Correlations between portal pressure and PTBF (A), and PTVR (B), in CBDL-8wk.

extremely strong linear correlation between portal pressure and HCVR was seen in Sham rats, this relationship was disproportionate in CBDL-4wk rats and was inverted in CBDL-8wk rats (Fig. 2). There was a significant negative correlation between PTBF and the hemoglobin concentration in CBDL-4 wh rats (Fig. 3), but this relationship was not significant in CBDL-8wk rats $(r=-0.420, p=0.300$, $n=8)$

\section{(4) Renal Circulation}

In both CBDL groups, renal artery blood flow (RABF) was significantly higher and renal artery vascular resistance (RAVR) was significantly lower than in Sham rats. There were no significant differences in these parameters between the CBDL- 
A

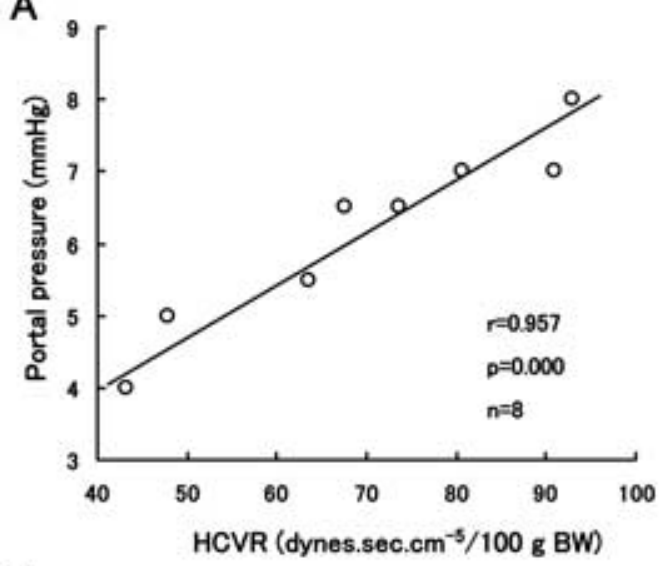

C

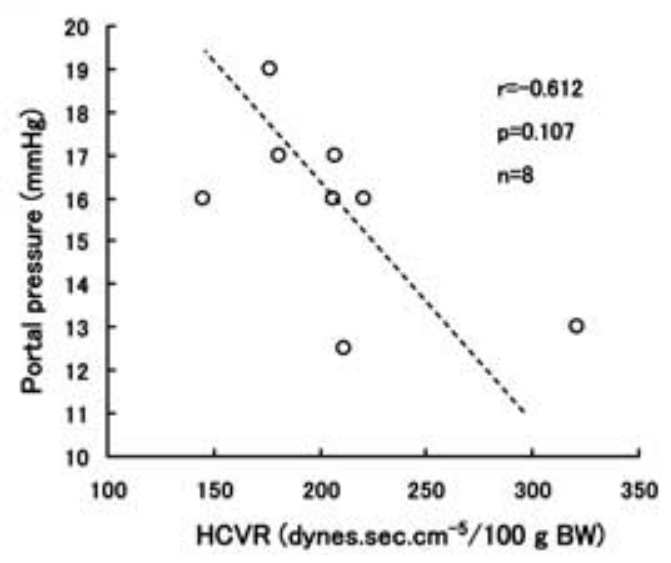

B

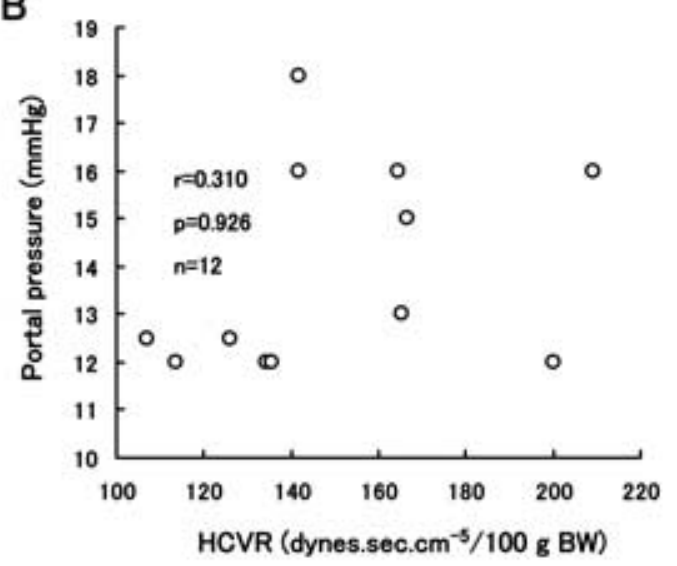

Fig. 2 Correlation between HCVR and portal pressure, in Sham rats (A), CBDL-4wk (B) and CBDL-8wk (C).

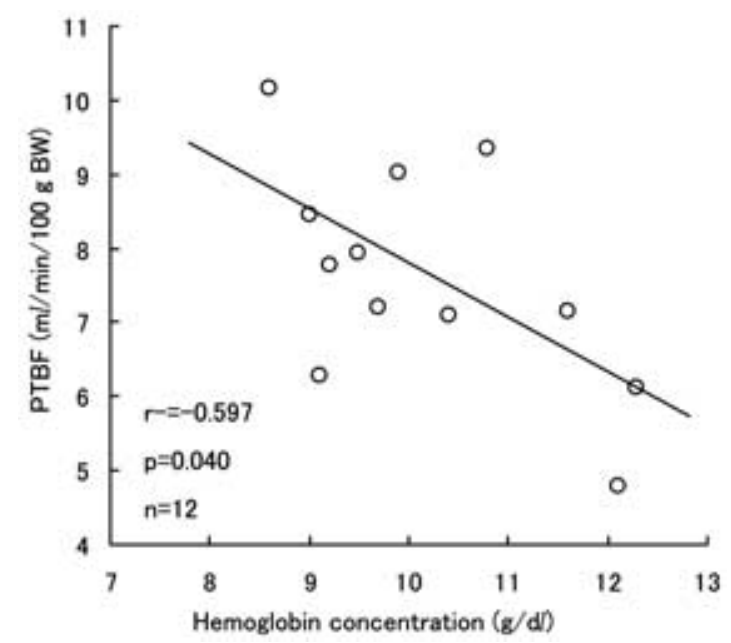

Fig. 3 Correlation between hemoglobin concentration and PTBF in CBDL-4wk.

8 wk and CBDL-4wk rats. Interestingly, RABF was significantly correlated with portal pressure and PTBF in the CBDL-8wk rats, but not in CBDL-4wk rats $(r=-0.453, p=0.139, n=13)$ or Sham rats $(r=$
-0.484, $\mathrm{p}=0.225, \mathrm{n}=8$ ) (Fig. 4). There was a significant positive correlation between RABF and CI in the CBDL-8wk group $(r=0.829, p=0.011, n=8)$, and in the Sham rats $(r=0.715, p=0.046, n=8)$. In the CBDL-4wk group, however, there were no significant correlations between RABF or RAVR and portal pressure or $\mathrm{CI}$.

\section{Discussion}

Portal pressure 8 weeks after CBDL was markedly increased in our model and was significantly higher than at 4 weeks, but the hyperdynamic systemic circulation that was prominent at 4 weeks became less evident by 8 weeks. The liver was hyperperfused at both 4 and 8 weeks, whereas PTBF was not increased at either time. Examination of the relationship between portal pressure and PTBF or PTVR suggested that the pressure rose along with an increase in PTBF and a 
A
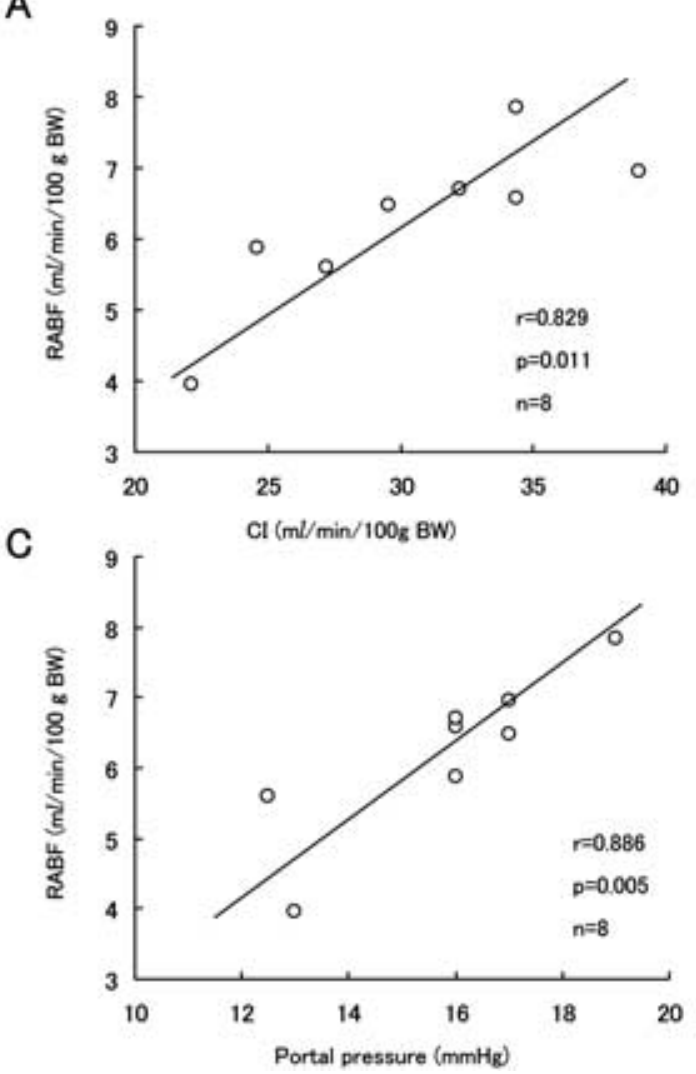

B

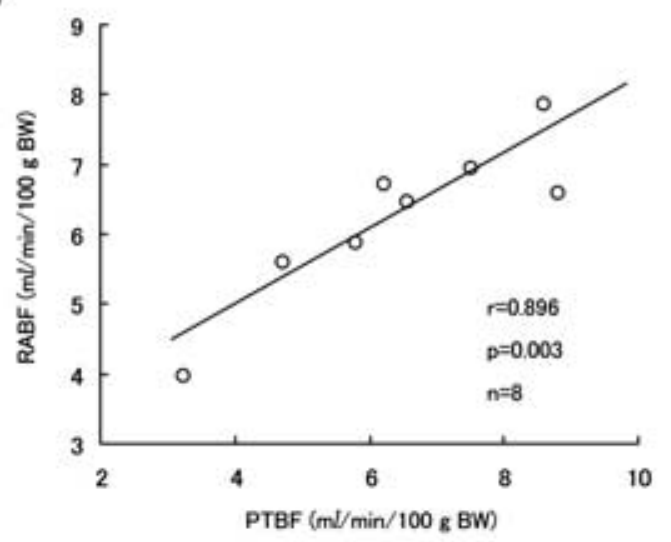

Fig. 4 Correlations between RABF and CI (A), RABF and PTBF (B), and RABF and portal pressure (C) in CBDL-8wk.

decrease in PTVR in the CBDL-8wk rats, indicating that elevation of the portal pressure was maintained by a mechanism corresponding to the forward flow theory of chronic portal hypertension ${ }^{4,715-17}$. Although backward flow, as represented by HCVR, was markedly increased in the CBDL-8wk rats, its correlation with the portal pressure was not significant (data not shown: $\mathrm{p}=0.107$, and $\mathrm{r}^{2}=0.375$ for the relative contribution of HCVR to the increment of portal pressure). In CBDL-4wk rats, the relationship between portal pressure and PTBF or PTVR was disproportionate and showed no significance. In PVL rats, it has been demonstrated that PTVR decreases with a resultant increase in $\mathrm{PTBF}^{4,7}$. Although a similar change was not prominent in our CBDL rats, HAVR decreased as $\mathrm{HABF}$ increased.

Splanchnic hyperemia and a hyperdynamic systemic circulation usually develop around 2 to 3 weeks after PVL, after which PTBF increases and PTVR decreases ${ }^{79,17}$. However, long-term follow-up of this portal hypertension model for 6 months has revealed that the hyperdynamic circulation becomes less prominent, although the portal pressure is still elevated $^{8,17}$. In rats with CBDL that survived beyond 8 weeks in the present study, the hyperdynamic circulation was also attenuated compared with that in CBDL-4wk rats. We have established a rabbit model with presinusoidal portal hypertension by injecting starch microspheres into the portal system $^{20}$. In this model, the portal pressure rises during the second week (consistent with the backward flow theory), declines in the fourth week, and rises again in the eighth week. The second increase in portal pressure is associated with an increase in portal blood flow, although portosystemic collateral flow also increases (consistent with the forward flow theory). These three kinds of longstanding portal hypertension models are similar with respect to the above points, supporting the forward flow theory.

Interestingly, a significant negative correlation between PTBF and hemoglobin concentration was observed in the CBDL-4wk group. The anemia that 
was seen 4 weeks after CBDL improved by 8 weeks after surgery. The cause of anemia and its recovery could not be determined in the present study (we confirmed the absence of internal hemorrhage at autopsy). It is difficult to attribute the anemia to hypersplenism, which is commonly associated with portal hypertension, because the portal pressure remained high at more than 8 weeks after the procedure. A previous study focusing on the relationship between hemodynamics and anemia revealed that a low hemoglobin concentration potentiated the hyperdynamic circulation in patients with cirrhosis and portal hypertension ${ }^{21-23}$. Conversely, an increase in hemoglobin concentration induced by administration of erythropoietin led to a reduction in splanchnic vasodilatation in rats with portal hypertension due to $\mathrm{PVL}^{23}$. This change was probably elicited by depletion of $\mathrm{NO}$ due to enhanced binding with hemoglobin ${ }^{23}$. The significant negative correlation between the hemoglobin concentration and the increase in $\mathrm{PTBF}$ in our CBDL-4wk rats, which had an increased serum level of NOx, appeared to be consistent with this hypothesis ${ }^{23}$. Even if the serum level of NOx, which has no effect on vascular smooth muscle, was increased in the CBDL-8wk group, the improvement in the hyperdynamic circulatory state (systemic and partly splanchnic) in these animals without anemia was also compatible with this hypothesis.

Particularly in the pulmonary vascular bed, it has been demonstrated that increased hepatic production and release of ET-1 is one mechanism triggering vasodilatation and an increase in pulmonary endothelial nitric oxide synthase levels after $\mathrm{CBDL}^{24,25}$. These changes are associated with a selective increase in pulmonary vascular ET-B receptor expression in patients with cirrhosis and portal hypertension, an event that appears to enhance ET-B receptor-mediated, ET-1-induced NO production. although our CBDL model also showed significant increases in plasma ET-1 and serum NOx, we could not find any significant correlation between ET-1 and NOx levels in either model.

Renal blood flow usually decreases in patients with advanced cirrhosis and portal hypertension ${ }^{26-28}$. In hepatorenal syndrome, severe ischemia of the renal cortex due to excessive vasoconstriction has been demonstrated ${ }^{27-29}$. Our CBDL-8wk rats did not show a decrease in renal blood flow and did not show features (extreme splanchinic hyperemia with moderate hypotension and marked ascites) necessary for the development of renal failure, such as hepatorenal syndrome. In an experimental animal model, it was found that renal hyperemia is associated with established portal hypertension due to PVL or cirrhosis induced by administration of $\mathrm{CCl}_{4}{ }^{10.30}$. Our rats with CBDL also showed renal hyperemia at an early stage, as well as in a late stage. In CBDL-8wk rats, it was interesting that $\mathrm{RABF}$ increased along with $\mathrm{CI}$ and portal pressure. It has been suggested that RABF is regulated by intrarenal, hormonal, and neural factors ${ }^{27,28,31-33}$. It was difficult to assess the mechanisms related to modulation of the renal circulation in the present study. However, the striking linear correlation between RABF and PTBF suggests that blood flow to both vascular beds might be regulated by the same factor or factors.

Although the portal pressure was high in rats surviving beyond 8 weeks, the systemic circulation was less hyperdynamic than in the early period after CBDL. Moreover, the rise in portal pressure appeared to be dependent on the increase in splanchnic blood flow or the decrease in splanchnic vascular resistance. This supports the forward flow theory as the mechanism maintaining chronic portal hypertension after the development of portal collateral channels. In our portal hypertension model created by CBDL, the hemodynamic abnormalities changed over time. It was suggested that a stable relationship among hemodynamic parameters was achieved in the advanced stage of CBDL and that the presence of anemia contributed to the lack of such stability in the early period after CBDL.

\section{References}

1. Lee SS, Girod C, Braillon A, Hadengue A, Lebrec D: Hemodynamic characterization of chronic bile ductligated rats: effect of pentobarbital sodium. Am J Physiol 1986; 251: G176-180.

2. Lee FY, Colombato LA, Albillos A, Groszmann RJ: Administration of $\mathrm{N}$ omega-nitro-L-arginine 
ameliorates portal-systemic shunting in portalhypertensive rats. Gastroenterology 1993; 105: 14641470.

3. Akimoto T, Hayashi N, Adachi M, Kobayashi N, Zhang XJ, Ohsuga M, Katsuta Y: Viability and plasma vitamin $\mathrm{K}$ levels in the common bile ductligated rats. Exp Anim 2005; 54: 155-161.

4. Sikuler E, Groszmann RJ: Interaction of flow and resistance in maintenance of portal hypertension in a rat model. Am J Physiol 1986; 250: G205-212.

5. Groszmann RJ, Vorobioff J, Riley E: Splanchnic hemodynamics in portal-hypertensive rats: measurement with gamma-labeled microspheres. Am J Physiol 1982; 242: G156-160.

6. Zhang XJ, Katsuta Y, Akimoto T, Ohsuga M, Aramaki T, Takano T: Intrapulmonary vascular dilatation and nitric oxide in hypoxemic rats with chronic bile duct ligation. J Hepatol 2003; 39: 724-730.

7. Sikuler E, Kravetz D, Groszmann RJ: Evolution of portal hypertension and mechanisms involved in its maintenance in a rat model. Am J Physiol 1985; 248 : G618-625.

8. Sikuler E, Groszmann RJ: Hemodynamic studies in long- and short-term portal hypertensive rats: the relation to systemic glucagon levels. Hepatology 1986; 6: 414-418.

9. Blanchet L, Lebrec D: Changes in splanchnic blood flow in portal hypertensive rats. Eur J Clin Invest 1982; 12: 327-330.

10. Premen AJ, Go VL, Banchs V, Benoit JN, Kvietys PR, Granger DN: Renal hyperemia in portal hypertension is not mediated by gastrointestinal peptides. Regul Pept 1986; 16: 39-49.

11. Kontos HA, Shapiro W, Mauck HP, Patterson JL Jr: General and regional circulatory alterations in cirrhosis of the liver. Am J Med 1964; 37: 526-535.

12. Gupta TK, Chen L, Groszmann RJ: Pathophysiology of portal hypertension. Clin Liver Dis 1997; 1: 1-12.

13. Gupta TK, Chen L, Groszmann RJ: Pathophysiology of portal hypertension. Baillieres Clin Gastroenterol 1997; 11: 203-219.

14. Bosch J, Pizcueta P, Feu F, Fernandez M, GarciaPagan JC: Pathophysiology of portal hypertension. Gastroenterol Clin North Am 1992; 21: 1-14.

15. Tsai YT, Lin HC, Lee SD: Recent advances in the pathophysiology of portal hypertension . J Gastroenterol Hepatol 1997; 12: S283-287.

16. Mo Vorobioff J, Bredfeldt JE, Groszmann RJ: Hyperdynamic circulation in portal-hypertensive rat model: a primary factor for maintenance of chronic portal hypertension. Am J Physiol 1983; 244: G52-57.

17. Benoit JN, Womack WA, Hernandez L, Granger DN: "Forward" and "backward" flow mechanisms of portal hypertension. Relative contributions in the rat model of portal vein stenosis. Gastroenterology 1985; 89: 1092-1096.

18. Green LC, Wagner DA, Glogowski J, Skipper PL, Wishnok JS, Tannenbaum SR: Analysis of nitrate, nitrite, and $[15 \mathrm{~N}]$ nitrate in biological fluids. Anal Biochem 1982; 126: 131-138.

19. Ando K, Hirata Y, Shichiri M, Emori T, Marumo F:
Presence of immunoreactive endothelin in human plasma. FEBS Lett 1989; 245: 164-166.

20. Komeichi H, Katsuta Y, Aramaki T, Okumura H: A new experimental animal model of portal hypertension. Intrahepatic portal obstruction by injecting DEAE-cross-linked dextran microspheres into the portal vein in the rabbit. Nippon Ika Daigaku Zasshi 1991; 58: 273-284.

21. Yang YY, Lin HC, Lee WC, Huang YT, Hou MC, Lee FY, Chang FY, Lee SD: Plasma erythropoietin level in patients with cirrhosis and its relationship to the severity of cirrhosis and renal function. J Gastroenterol Hepatol 2003; 18: 1156-1161.

22. Cirera I, Elizalde JI, Pique JM, Feu F, Casadevall M, Goldin E, Teres J, Bosch J, Rodes J: Anemia worsens hyperdynamic circulation of patients with cirrhosis and portal hypertension. Dig Dis Sci 1997; 42: 16971702.

23. Luo B, Abrams GA, Fallon MB: Endothelin-1 in the rat bile duct ligation model of hepatopulmonary syndrome: correlation with pulmonary dysfunction. J Hepatol 1998; 29: 571-578.

24. Zhang M, Luo B, Chen SJ, Abrams GA, Fallon MB: Endothelin-1 stimulation of endothelial nitric oxide synthase in the pathogenesis of hepatopulmonary syndrome. Am J Physiol 1999; 277 (5 Pt 1): G944-952.

25. Casadevall M, Pique JM, Cirera I, Goldin E, Elizalde I, Panes J, Martinez-Cuesta MA, Bosch J, Teres J, Rodes J: Increased blood hemoglobin attenuates splanchnic vasodilation in portal-hypertensive rats by nitric oxide inactivation. Gastroenterology 1996; 110: $1156-1165$.

26. Gines P, Guevara M, Arroyo V, Rodes J: Hepatorenal syndrome. Lancet 2003; 362: 1819-1827.

27. Gines P, Fernandez-Esparrach G, Arroyo V, Rodes J: Pathogenesis of ascites in cirrhosis. Semin Liver Dis 1997; 17: 175-189.

28. Epstein M, Berk DP, Hollenberg NK, Adams DF, Chalmers TC, Abrams HL, Merrill JP: Renal failure in the patient with cirrhosis. The role of active vasoconstriction. Am J Med 1970; 49: 175-185.

29. Sacerdoti D, Merlo A, Merkel C, Zuin R, Gatta A: Redistribution of renal blood flow in patients with liver cirrhosis. The role of renal PGE2. J Hepatol 1986; 2: 253-261.

30. Fernandez-Munoz D, Caramelo C, Santos JC, Blanchart A, Hernando L, Lopez-Novoa JM: Systemic and splanchnic hemodynamic disturbances in conscious rats with experimental liver cirrhosis without ascites. Am J Physiol 1985; 249: G316-320.

31. Cardenas A: Hepatorenal syndrome: a dreaded complication of end-stage liver disease. Am J Gastroenterol 2005; 100: 460-467.

32. Moreau R: Hepatorenal syndrome in patients with cirrhosis. J Gastroenterol Hepatol 2002; 17: 739-747.

33. Wong F, Blendis L: New challenge of hepatorenal syndrome: prevention and treatment. Hepatology 2001; 34: 1242-1251.

(Received, April 6, 2005)

(Accepted, June 8, 2005) 